\title{
Efeitos de refinamentos $h$ e $p$ para subespaços de funções do tipo Hdiv em malhas irregulares
}

\author{
Denise de Siqueira \\ Departamento de Matemática, UTFPR \\ 80230-901, Curitiba, PR \\ E-mail: denisesiqueira@uftpr.edu.br \\ Philippe R.B. Devloo \\ Faculdade de Engenharia Civil, FEC, Unicamp \\ 13083-552, Campinas, SP \\ E-mail:phil@fec.unicamp.br \\ Sônia M. Gomes \\ Instituto de Matemática, Estatística e Computação Científica, IMECC, Unicamp \\ 13083-859, Campinas, SP \\ E-mail: soniag@ime.unicamp.br.
}

\begin{abstract}
Resumo: É sabido que o emprego de formulações mistas é mais apropriado para simulações de fluxos em meios não homogêneos do que o modelo clássico em termos apenas da pressão. Tais formulações tem como característica a aproximação simultânea dos campos de pressão e velocidade, podendo fornecer boas soluções numéricas para esses campos ao mesmo tempo em que garante a conservação local de massa. No entanto para adotar tal estratégia é necessária a construção de bases para subespaços de elementos finitos do tipo Hdiv para o fluxo que sejam balanceados com subespaços de elementos finitos para a pressão. Em [2] foi apresentada uma sistemática de construção de bases para subespaços de Hdiv usando funções vetoriais polinomiais de grau uniforme em malhas triagulares e quadrilaterais. O presente trabalho tem como objetivo apresentar o desempenho das aproximações construídas na presença de refinamentos $h$ e $p$ em malhas irregulares.
\end{abstract}

Palavras-chave: Formulações Mistas, Espaços Hdiv, Refinamentos h e p, Malhas irregulares

\section{Introdução}

Os métodos mistos são conhecidos por utilizarem mais de um espaço de aproximação em sua formulação. Tal técnica é adotada quando se deseja aproximar simultaneamente duas ou mais variáveis de interesse. Se considerarmos um problema elíptico, como o descrito por um problema de Darcy, podemos fazer uso dos métodos mistos com o intuito de se aproximar, simultaneamente, as variáveis de pressão e velocidade. Para um bom desempenho deste tipo de abordagem é necessário um balanceamento entre os espaços envolvidos. No caso do problema de Darcy, procura-se que o espaço de aproximação associado à variável de fluxo (espaço HDiv) e o espaço de aproximação associado à pressão, sejam balanceados.

Em [2] foi estabelecida uma sistemática para construção de funções de base bidimensionais para subespaços de elementos finitos $V_{h} \subset$ Hdiv. A sistemática consiste em multiplicar funções escalares $\varphi$ do tipo $H^{1}$, desenvolvidas em [1], por vetores $\mathbf{v}$, convenientemente construídos, gerando funções vetoriais $\mathbf{u}=\varphi \mathbf{v}$. 
A particularidade desta construção está no fato de que o campo vetorial é construído baseando-se na geometria de cada elemento da partição, não no elemento mestre (ou de referência), o que evita problemas com orientação de vetores. As características de conformidade para funções de $H^{1}$ das funções de base $\varphi$, em conjunto com os aspectos específicos do campo vetorial, possibilitam a validação da condição fundamental dos espaços do tipo Hdiv que é a continuidade da componente normal nas interfaces dos elementos.

Uma vez criadas funções de base para subespaços do tipo HDiv, é necessário estudar seu balanceamento com o espaço de aproximação associado à variável de pressão. Os testes numéricos apresentados em [2] mostram que, para malhas triangulares uniformes, um tipo de balanceamento é conseguido se a ordem polinomial $k$ adotada para um subespaço de aproximação Hdiv para a variável de fluxo for uma ordem a mais se comparada com aproximações em $L^{2}$ para variável de pressão. Em tal caso, os pares de espaços de aproximação são denotados por $\mathcal{P}_{k} \mathcal{P}_{k-1}$. Para malhas quadrilaterais, é necessário enriquecer o espaço de fluxo com algumas funções, convenientemente escolhidas, de modo a manter o balanceamento entre os espaços. Adotando-se ordem $k$ para pressão, para o subespaço associado ao fluxo acrescentam-se algumas funções de ordem $k+1$ associados aos graus de liberdade internos ao elemento, denotados por $\mathcal{Q}_{k}^{+} \mathcal{Q}_{k}$. O objetivo do presente estudo é verificar se esse balanceamento se mantém no contexto de malhas não uniformes com refinamentos dos tipos $h$ e $p$.

\section{Problema modelo}

Considera-se o problema modelo de encontrar $p$ tal que

$$
\left\{\begin{array}{cccc}
\triangle p & = & f, & \text { em } \Omega=[0,1]^{2} \\
p & = & 0 & \text { em } \partial \Omega
\end{array}\right.
$$

cuja solução analítica é dada por

$$
\begin{aligned}
& p(x, y)=\sin (\pi x) \cos (\pi y) \\
& \mathbf{u}(x, y)=-\nabla p=(-\cos (\pi x) \cos (\pi y), \sin (\pi y) \sin (\pi x))^{T} .
\end{aligned}
$$

A formulação mista associada a tal problema é dada por: Encontrar $(\mathbf{u}, p) \in H \operatorname{div} \times L^{2}$ tal que

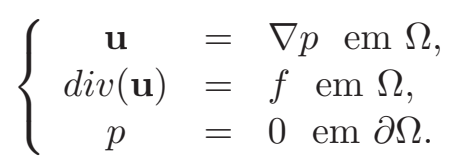

Considerando $V_{h} \subset \mathrm{H}\left(\right.$ div) e $Y_{h} \subset L^{2}$ subespaços de aproximação, o problema discreto associado a (2) consiste em: Encontrar $\left(\mathbf{u}_{h}, p_{h}\right) \in V_{h} \times Y_{h}$ tal que

$$
\left\{\begin{aligned}
a\left(\mathbf{u}_{h}, \mathbf{v}_{h}\right)+b\left(\mathbf{v}_{h}, p_{h}\right) & =0 \forall \mathbf{v}_{h} \in V_{h} \\
b\left(\mathbf{v}_{h}, w_{h}\right) & =f_{h} \forall w_{h} \in Y_{h}
\end{aligned}\right.
$$

Consideram-se subespaços baseados em dois tipos de malhas, uniforme e não uniforme. Para as malhas não uniformes, o padrão de refinamento adotado é ilustradado na Figura 1.

\section{Experimentos Numéricos}

Os experimentos numéricos apresentados a continuação foram implementados no NeoPZ (www.labmec.org.br/wiki/neopz/start), uma plataforma de código aberto, formada por um conjunto de classes, idealizadas para permitir o desenvolvimento de códigos de elementos finitos com muita flexibilidade, permitindo refinamentos do tipo $h p$, em que foram incorporados os espaços de aproximação do tipo Hdiv mencionados. 


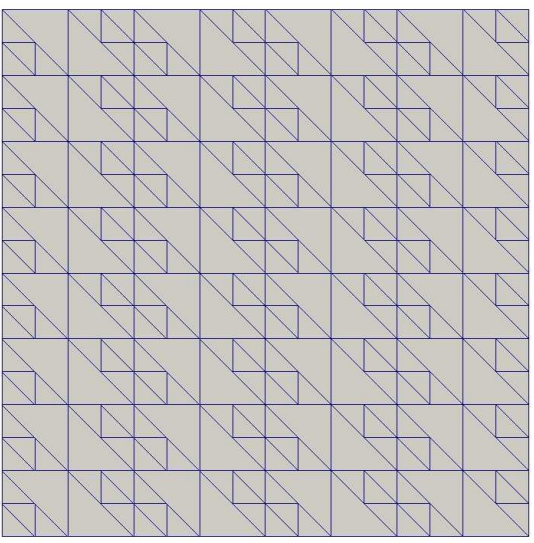

(a) Malha triangular

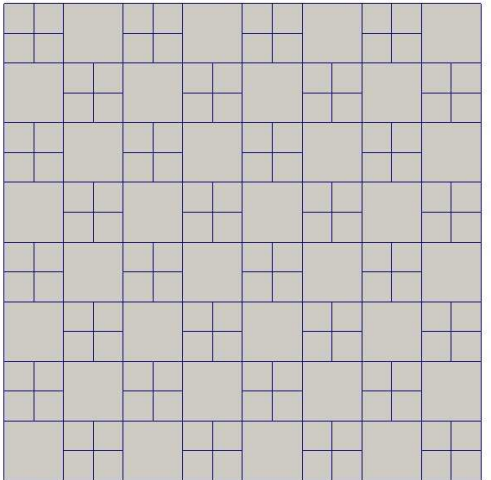

(b) Malha quadrilateral

Figura 1: Malhas não uniformes

A Figura 2 apresenta os resultados obtidos usando malhas triangulares com refinamento do tipo $h$, para diferentes ordens polinomiais. O gráfico apresenta a relação entre o $\log (h)$ pelo $\log \left(\right.$ erro), sendo $h$ o espaçamento da malha e o erro calculado na norma $L^{2}$. As linhas tracejadas indicam os resultados para malhas não uniformes, ou seja, para refinamentos cujo padrão é como ilustrado na Figura 1 (a), enquanto que as linhas contínuas indicam malhas uniformes.

É possível observar que a taxa de convergência para a variável de fluxo u é uma ordem a mais, se comparada com a da pressão $p$ e que as taxas de convergência não foram alteradas com a utilização de malhas não uniformes. Mais do que isso, observa-se que o erro nas aproximações com malhas triangulares não uniformes é um pouco menor se comparado com os resultados da malha regular.

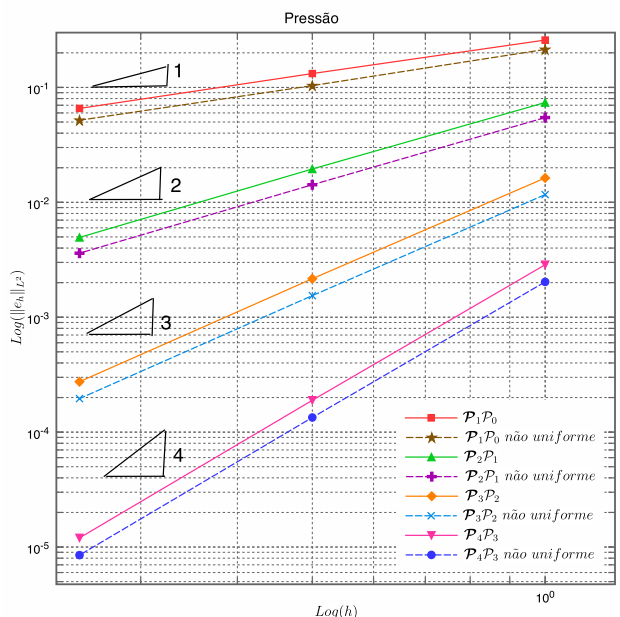

Aproximação da pressão

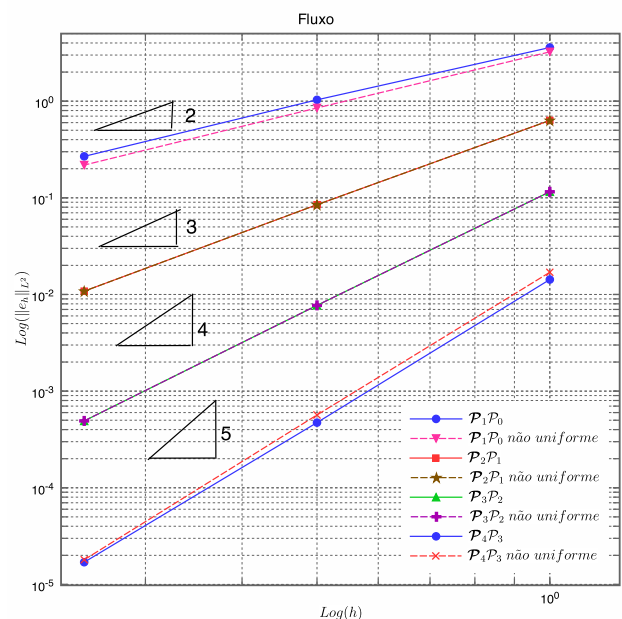

Aproximação do fluxo

Figura 2: Taxas de convergência para malhas triangulares com refinamento do tipo $h$

A Figura 3 apresenta os resultados obtidos com uma malha triangular com um refinamento uniforme na ordem polinomial, ou seja, mantém-se fixa a malha (uniforme ou não) e varia-se a ordem polinomial $k$ do elemento. O resultado apresentado mostra a relação, em escala logarítimica, 
do número de graus de liberdade pela norma $L^{2}$ do erro. Observa-se um comportamento do tipo exponencial, como esperado para este tipo de refinamento.

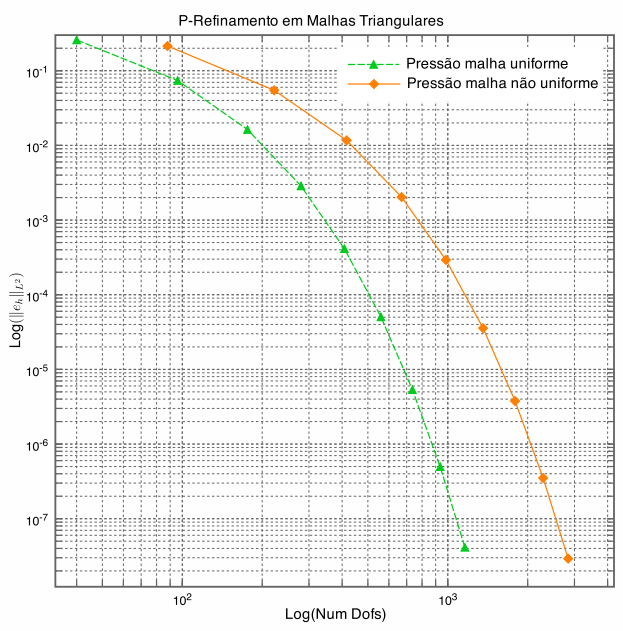

Aproximação da pressão

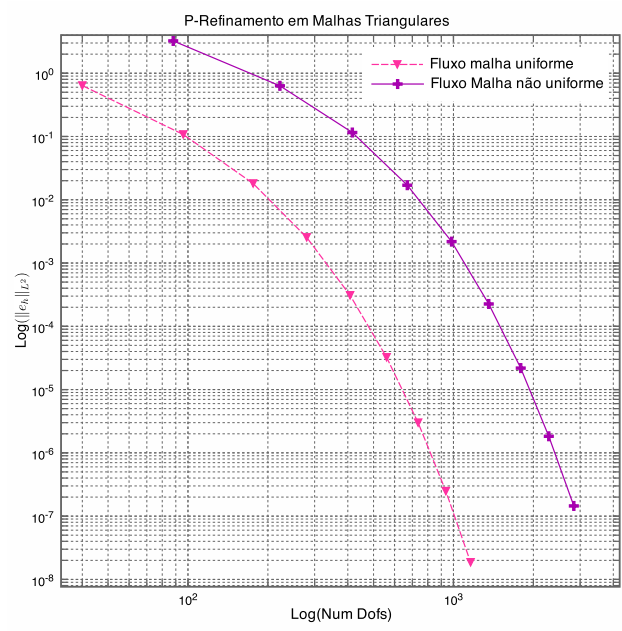

Aproximação do fluxo

Figura 3: Taxas de convergência para malhas triangulares com refinamento na ordem polinomial.

A Figura 4 mostra as taxas de convergência utilizando malhas quadrilaterias. Assim como no caso triangular, as linhas tracejadas indicam o uso de malhas não uniformes enquanto que linhas contínuas indicam malhas uniformes.

De acordo com o gráfico, é possível observar que as taxas de convergência para fluxo e pressão são de mesma ordem. Tais resultados se justificam uma vez que o fluxo é aproximado por funções polinomiais de ordem $k$ enriquecidas apenas com algumas funções de ordem $k+1$. Observa-se ainda que, mesmo usando malhas não uniformes, a taxa de convergência mantém-se a mesma, sendo possível ainda observar que o erro nas malhas não uniformes é ligeiramente menor se comparado com as malhas uniformes.

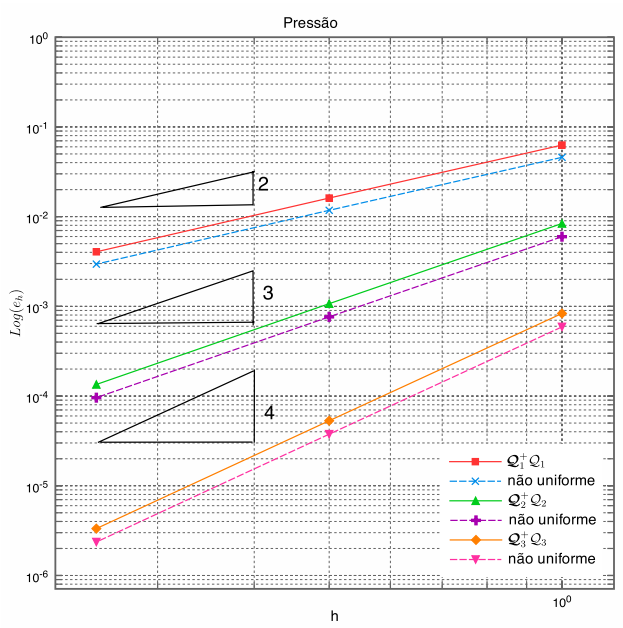

Taxa de convergência para a pressão

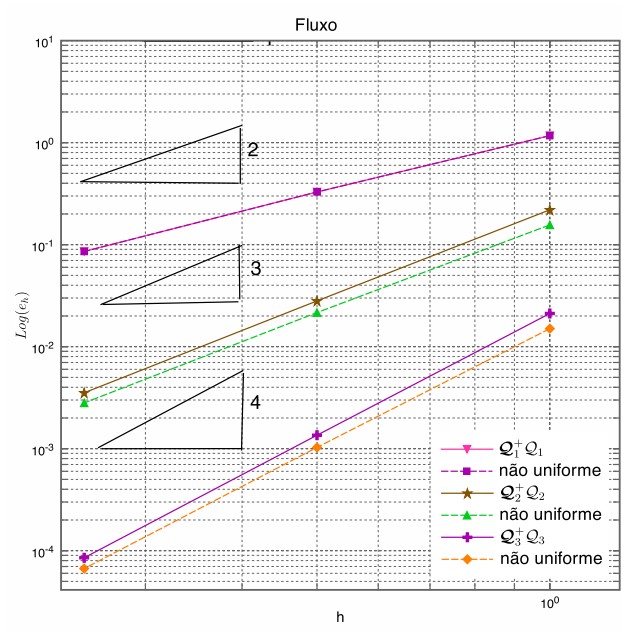

Taxa de convergência para o fluxo

Figura 4: Taxas de convergência para malhas quadrilaterais com refinamento do tipo $h$ 


\section{Conclusão}

Foi implementado um $h$ refinamento em malhas uniformes e não uniformes para espaços de aproximação para a formulação mista e foi observado que tanto para malhas quadrilaterias quanto triangulares a taxa de convergência manteve-se a mesma, o que mostra a consistência do refinamento $h$ para os subespaços adotados.

Observou-se ainda que, tanto para o fluxo quanto para a pressão, o erro absoluto nas aproximações baseadas em malhas quadrilaterias (com refinamento uniforme ou não) é menor se comparados com as malhas triangulares.

Aplicou-se ainda testes para verificar a consistência dos subespaços desenvolvidos com relação ao refinamento da ordem polinomial. Os resultados numéricos mostram um decaimento do erro na norma $L^{2}$ do tipo exponencial, de acordo com o apresentado na literatura.

Na continuação, pretende-se avaliar também o comportamento de tais subespaços quando uma distribuição não uniforme das ordens de aproximação é utilizada (refinameno do tipo $h p$ ) .

\section{Referências}

[1] Bravo,C. M. A. A.,Devloo, P. R. B., Rylo, E.C., Systematic and generic construction of shape functions for p-adaptive meshes of multidimensional finite elements, Comp. Met. Appl. Mech. Eng. 198 (2009) 1716-1725.

[2] Siqueira, D., Devloo, P. R. B., Gomes, S.M., Hierarchical high order finite element approximation spaces for Hdiv and Hcurl, in: G. Kreiss, P. Lötstedt, Malqvist A., M. Neytcheva (Eds.), Numerical Mathematics and Advanced Applications, Springer, Upsalla, 2009, pp. 267-274.

[3] Siqueira, D. ; Devloo, P.R.B. ; Gomes, M. Sonia . A new procedure for the construction of hierarchical high order Hdiv and Hcurl finite element spaces. Journal of Computational and Applied Mathematics, v. 240, p. 204-214, 2013. 